\title{
In-flight calibration of double-probe electric field measurements on Cluster
}

\author{
Y. V. Khotyaintsev ${ }^{1}$, P.-A. Lindqvist ${ }^{2}$, C. M. Cully ${ }^{1, *}$, A. I. Eriksson ${ }^{1}$, and M. André ${ }^{1}$ \\ ${ }^{1}$ Swedish Institute of Space Physics, Uppsala, Sweden \\ ${ }^{2}$ Royal Institute of Technology, Stockholm, Sweden \\ *now at: Department of Physics and Astronomy, University of Calgary, Calgary, Canada
}

Correspondence to: Y. V. Khotyaintsev (yuri@irfu.se)

Received: 19 August 2013 - Published in Geosci. Instrum. Method. Data Syst. Discuss.: 31 January 2014

Revised: 3 July 2014 - Accepted: 4 August 2014 - Published: 27 August 2014

\begin{abstract}
Double-probe electric field instrument with long wire booms is one of the most popular techniques for in situ measurement of electric fields in plasmas on spinning spacecraft platforms, which have been employed on a large number of space missions. Here we present an overview of the calibration procedure used for the Electric Field and Wave (EFW) instrument on Cluster, which involves spin fits of the data and correction of several offsets. We also describe the procedure for the offset determination and present results for the long-term evolution of the offsets.
\end{abstract}

\section{Introduction}

Double-probe electric field experiments have been flown on a number of spacecraft (see review by Pedersen et al., 1998) including Cluster (Gustafsson et al., 1997, 2001), and calibration of the direct-current (DC) electric field has always been a challenging and time-consuming task. Main reasons for this are strong influence of the ambient plasma and the spacecraft itself on the measurements. Other techniques to measure electric fields at low time resolution as electron drift instruments and ion spectrometers, such as Electron Drift Instrument (EDI) (Paschmann et al., 1997, 2001) and Cluster Ion Spectrometry (CIS) (Rème et al., 2001) on Cluster, are immune to some of the problems affecting the double-probe measurements, but have their own limitations. With the Cluster Active Archive a semi-automatic approach to in-flight calibration of the DC electric field data has been developed (Khotyaintsev et al., 2010), and the purpose of this paper is to describe the main elements of this calibration procedure under nominal operations.

An example of a clear deviation from nominal performance is the non-geophysical electric field detected by Electric Field and Wave experiment (EFW) due to the wake behind the spacecraft caused by cool $(\mathrm{eV})$ outflowing ionospheric ions drifting at supersonic velocities (Eriksson et al., 2006). Such ions are common in the magnetospheric tail lobes. Careful investigation of this "problem" has resulted in a new method to detect positive low-energy ions, otherwise invisible to detectors on a sunlit spacecraft positively charged to several volts (Engwall et al., 2006, 2009a, b; André and Cully, 2012). However, it is not possible to recover the ambient geophysical electric field in such cases.

\section{Short instrument description}

The detector of the Cluster EFW instrument consists of four spherical sensors numbered 1 to 4 deployed orthogonally on $42.5 \mathrm{~m}$ long wire booms in the spin plane of the spacecraft (see Fig. 1). The spacecraft makes one rotation in approximately $4 \mathrm{~s}$. The potential drop between two sensors, separated by $88 \mathrm{~m}$ (or $62 \mathrm{~m}$ in case of using non-opposing probes) tip to tip, is measured to provide an electric field measurement. The probe difference signals are normally routed through $10 \mathrm{~Hz}$ anti-aliasing low-pass filters when sampled at $25 \mathrm{~s}^{-1}$, and through $180 \mathrm{~Hz}$ low-pass filters when sampled at $450 \mathrm{~s}^{-1}$. The potential difference between each sensor and the spacecraft is measured separately with a sampling frequency of $5 \mathrm{~s}^{-1}$ after routing through low-pass filters with 


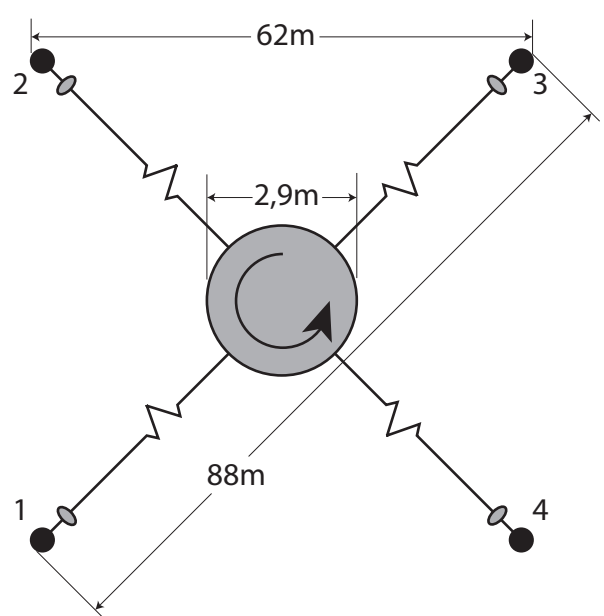

Figure 1. Cluster EFW double-probe electric field instrument.

a cut-off frequency of $10 \mathrm{~Hz}$. A detailed description of the EFW instrument can be found in Gustafsson et al. (1997, 2001).

\section{Calibration procedure}

The goal of the calibration procedure is to obtain geophysical DC electric field in the spacecraft spin plane in a despun reference frame. On Cluster we used the ISR2 (Inverted Spin Reference) system, also known as DSI (Despun System Inverted). The $x$ and $y$ axes are in the spin plane, with $x$ axis pointing as near sunward as possible and $y$ axis perpendicular to the sunward direction, positive towards dusk. The $z$ axis is along the (negative) spacecraft spin axis, towards the north ecliptic. The coordinate system is called "inverted" because the actual spin axis of Cluster is pointing towards the south ecliptic. The ISR2 system thus is identical to Geocentric Solar Ecliptic system (GSE) if the satellite spin axis angle to ecliptic north is zero, and is a good approximation to GSE for the usual case of this angle being a few degrees.

\subsection{Raw data}

The raw data available from EFW under normal circumstances are the two orthogonal electric field components in the spinning frame ( $E_{12}$ and $\left.E_{34}\right)$ sampled at 25 or $450 \mathrm{~Hz}$, as well as potentials of the individual probes $\left(P_{1}, P_{2}, P_{3}\right.$ and $P_{4}$ ) sampled at $5 \mathrm{~Hz}$. In case of probe 1 failure (for dates of permanent failures on $\mathrm{C} 1, \mathrm{C} 2$ and $\mathrm{C} 3$, see Lindqvist et al., 2013), instead of E12 we use E32. An example of raw data is shown in Fig. 2.

As the first stage of calibration, it is necessary to perform initial cleaning of the data at which we remove intervals with bad data due to issues with electronics, probe saturations due to low plasma density (often occurring in in the magnetospheric lobes), and saturations due to non-optimal

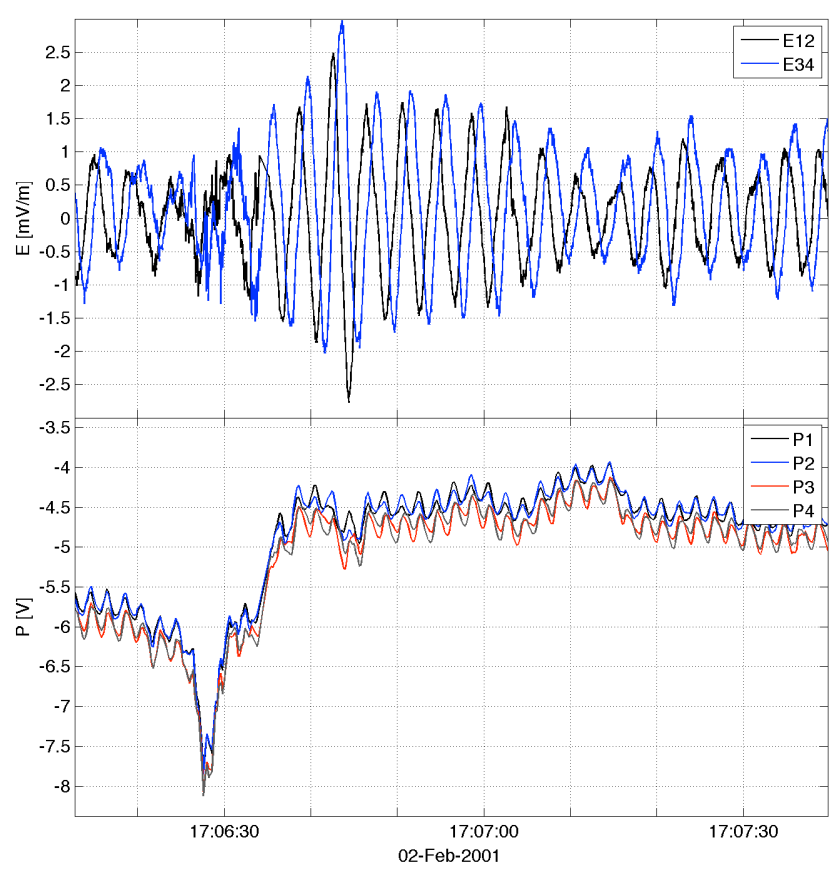

Figure 2. Raw data of the electric field, $E_{12}, E_{34}$ (upper panel) and of the probe-to-spacecraft potential $P_{1}, P_{2}, P_{3}, P_{4}$ (bottom panel) measured by Cluster 1 .

bias current settings occurring in dense plasmas such as magnetosheath and plasmasphere (Khotyaintsev et al., 2010). If the spacecraft is in the solar wind, we apply a correction for the wakes usually present in the raw data (Eriksson et al., 2007).

\subsection{Spin fits}

After initial cleaning of the data, a spin fitting procedure is performed; the output of this procedure provides basic parameters that are used later in the calibration procedure. In the presence of a constant ambient electric field, the raw data signal (probe potential difference) is a sine wave (see Fig. 2, upper panel) where the amplitude and phase give the electric field magnitude and direction. A least-squares fit to the raw data of the form

$$
\begin{aligned}
y & =A+B \sin (\omega t)+C \cos (\omega t)+D \sin (2 \omega t) \\
& +E \cos (2 \omega t)+\ldots
\end{aligned}
$$

is done once every $4 \mathrm{~s}(2 \pi / \omega \approx 4 \mathrm{~s}$ is approximately the spacecraft spin period) and the fit is applied to $4 \mathrm{~s}$ long time intervals.

The standard deviation of the raw data from the fitted sine wave can be used as an indication of high-frequency variations in the data. Higher order terms, $D, E, \ldots$, may be used for diagnostics of data quality: normally the higher order terms are much smaller than $B$ and $C$, and the opposite situation would indicate problems with the measurements. 


\subsection{Offsets}

The sine and cosine terms, $B$ and $C$, after correction for ISR2 offsets provide the $4 \mathrm{~s}$ (spin) resolution electric field in ISR2:

$E_{x 4 s}=\alpha\left(B-\Delta E_{x}\right)$,

$E_{y 4 s}=\alpha\left(C-\Delta E_{y}\right)$,

where $\alpha$ is the amplitude correction factor due to the ambient electric field being "short-circuited" by the presence of the spacecraft and wire booms (see Sect. 4.1). And $\Delta E_{x}$ (sunward offset) and $\Delta E_{y}$ (duskward offset) are the ISR2 offsets, which represent the difference between the measured and geophysical electric fields in the despun frame and are discussed in detail later.

As the spin fitting procedure would typically yield different values for the electric field from the two different probe pairs, it is useful to introduce an additional offset which describes the difference between the two measurements, $\Delta_{p 12 p 34}$, which we call the delta offset:

$$
\begin{aligned}
& \Delta_{x p 12 p 34}=E_{x 4 s}\left(E_{12}\right)-E_{x 4 s}\left(E_{34}\right), \\
& \Delta_{y p 12 p 34}=E_{y 4 s}\left(E_{12}\right)-E_{y 4 s}\left(E_{34}\right) .
\end{aligned}
$$

The despun full-resolution electric field is obtained as follows:

$E_{x}=\operatorname{Re}\left[\varepsilon_{12}\right]-\Delta_{x p 12 p 34}+\operatorname{Re}\left[\varepsilon_{34}\right]$,

$E_{y}=\operatorname{Im}\left[\varepsilon_{12}\right]-\Delta_{y p 12 p 34}+\operatorname{Im}\left[\varepsilon_{34}\right]$,

where $\varepsilon_{12}=\left(E_{12}-\Delta_{\text {raw } 12}\right) e^{i \phi 12}, \varepsilon_{34}=\left(E_{34}-\Delta_{\text {raw } 34}\right)$ $e^{i \phi 34}$, and $\phi_{12}=\phi_{34}+\pi / 2$ is the spin phase of probe 1 with respect to the sun; raw data $D C$ offset, $\Delta_{\text {raw }}=\langle A\rangle$, is based on parameter $A$ of the fit (Eq. 1). Ideally, the DC level of the raw data should be zero. However, small differences between the probe surfaces and in the electronics create a DC offset in the raw data. If not corrected, it shows up as a signal at the spin frequency in the despun electric field.

It must be noted that asymmetries due to the direction to the sun have the dominant contribution to the offsets, so that the following inequalities are typically satisfied:

$$
\begin{aligned}
& \Delta E_{x} \gg \Delta E_{y}, \\
& \Delta_{x p 12 p 34} \gg \Delta_{y p 12 p 34} .
\end{aligned}
$$

\section{Results}

In this section we summarize the main results concerning the various offsets defined above. Raw data DC offset and delta offsets are obtained from spin fits, while the amplitude correction factor and ISR2 offsets are obtained based on interspacecraft calibration as well as cross-calibration with CIS (Rème et al., 2001) and EDI (Paschmann et al., 1997, 2001).

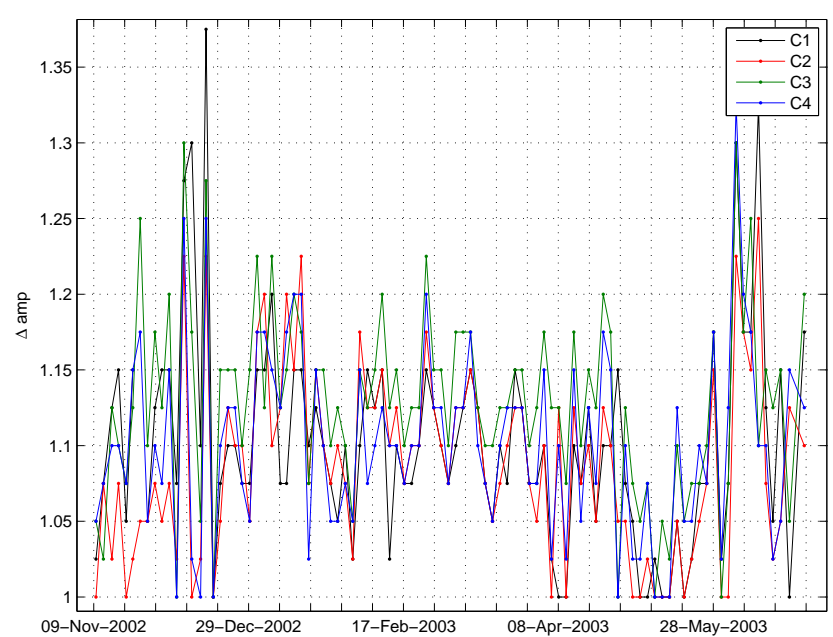

Figure 3. Amplitude correction factor for the electric field measured by EFW on Cluster 1-4 during a solar wind season from November 2002 to June 2003.

\subsection{Amplitude factors}

Amplitude factors are needed since the electric field is partially "short circuited" by the spacecraft potential, which is also the potential of the wire booms, extending out to a large distance from the spacecraft (Cully et al., 2007).

We have used the ISR2 $y$ components of the electric field to determine the amplitude correction factor. This component of $E$ is generally free from offsets, and thus by comparing $E_{y}$ from EFW and CIS-HIA we are able to deduce the amplitude correction factor. Results of such computations for the spring season of 2002 are shown in Fig. 3. Every point in the plot corresponds to one orbit of data. One should mention that variations seen in the data are not caused by changes in the factor, but rather by "bad data" and insufficient data coverage.

On the basis of simulations and comparisons with other Cluster instruments, it has been determined that the measured electric field magnitude needs to be multiplied by a factor of $\alpha=1.1$. We use this constant value through the entire mission. This value is consistent with valued obtained from simulations of the spacecraft-plasma interaction (Cully et al., 2007).

\subsection{Raw data DC offset}

The raw data DC offset, $\Delta_{\text {raw }}$, from the both probe pairs is used to calculate the full-resolution E-field. It is applied to $E_{12}$ and $E_{34}$ prior to despinning. Variations in the electric field will result in small changes to $A$ computed from spin fits for different $4 \mathrm{~s}$ intervals. So if $\Delta_{\text {raw }}$ depended only on the electronics, one could compute a long-term average of $A$ and use it as $\Delta_{\text {raw }}$. But we find that $A$ also depends on the surrounding plasma environment as illustrated in Fig. 4, where $A$ is plotted as a function of the spacecraft potential. 


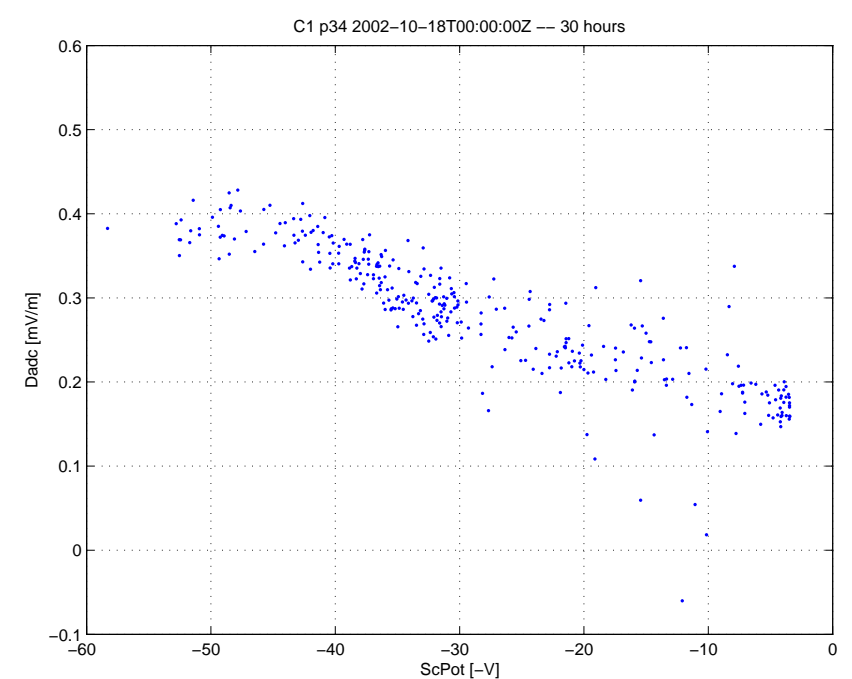

Figure 4. Dependence of the raw data DC offset, $\Delta_{\text {raw }}$, computed from the spin fits on the spacecraft potential showing that the offset decreases when the potential is close to zero (characteristic of dense plasmas).

Therefore we want a smoothened value and at the same time to track changes in the plasma environment. That is why the DC offset is smoothed, $\Delta_{\text {raw }}=\langle A\rangle$, using a weighted average over seven spins using weights $[0.07,0.15,0.18,0.2$, $0.18,0.15,0.07]$. This approach was selected based on an empirical basis after testing several different lengths of the averaging interval.

\subsection{ISR2 offsets}

Our main assumption in the study of ISR2 offsets is that the offsets depend on the instrument configuration, spacecraft attitude and that the dependence on surrounding plasma parameters is weak, i.e., being in the same kind of plasma environment (for example plasma sheet) and having the same instrument settings and probe properties for two different time intervals, and the difference between ISR 2 offsets for the two intervals must be within the uncertainty of the offset determination (fraction of $\mathrm{mV} \mathrm{m}^{-1}$ ). As the offsets still depend on the plasma environment, we decided to split the data set into two groups - "solar wind/magnetosheath" and "magnetosphere" - which correspond to two situations with "cold and dense" and "hot and rarefied" plasmas. To split every orbit into these two groups, we have used the Shue magnetopause model (Shue et al., 1997) with realistic solar wind parameters measured by the ACE spacecraft. For each of the groups we statistically determine offsets over a period of several weeks to several months in order to account for changes in the instrument setting, spacecraft attitude, solar UV flux, etc. Then, based on the position along the orbit, observed value of the spacecraft potential and manual inspection, we determine which of the two offsets is to be applied at each point.

\subsubsection{ISR2 offsets in the solar wind and magnetosheath}

For the solar wind/magnetosheath intervals, we first perform the inter-spacecraft calibration under assumption that all the spacecraft observe the same large-scale electric field, which is the case in the solar wind. As a result for each interval (from outbound magnetopause crossing to the inbound, typically several hours long), we get relative offset between the spacecraft, which are the differences in $E_{x}$ and $E_{y}$ between the different spacecraft averaged over the entire interval. Figure 5 shows an example of such an interval, and the two upper panels show $E_{x}$ and $E_{y}$ from all four spacecraft.

Then by using CIS-HIA from C1 and/or C3 as reference data, we find the ISR2 offsets for EFW for each of the spacecraft. We get one value for offsets per orbit. The procedure can be controlled visually by using a type of plot presented in Fig. 5. The two upper panels show all the available EFW and CIS-HIA data ( $E_{x}$ and $E_{y}$ in ISR2). Then we construct the reference E-field from CIS-HIA by averaging data from the spacecraft where CIS-HIA data are available. Such averaging is possible as the difference between the spacecraft in the solar wind/magnetosheath is typically small. Then we compute the difference between the EFW $E_{x}$ on all spacecraft and the reference E-field; this difference is plotted in the third panel. Average of the difference over the entire interval gives the local IRS2 offset. This offset is then applied to the EFW on different spacecraft. The resulted corrected and reference E-fields are plotted in the two bottom panels.

Evolution of ISR2 offsets in the solar wind and magnetosheath during mission lifetime is shown in Fig. 6. One can see that the offsets are rather steady and slowly decreasing with approach of the solar minimum $(\sim 2009)$. The only striking feature is the sudden increase of the offset in C3 in 2005. This change is not yet understood.

\subsubsection{ISR2 offsets in the magnetosphere}

The problem of determining the offsets in the magnetosphere is significantly more complicated in comparison to the solar wind/magnetosheath. Data from the other instruments, which could have been used as a reference, are of very low quality in large areas of the magnetosphere due to low counts (the CIS instrument) or low magnetic fields (the EDI instrument). Also the EFW data are subject to frequent problems, such as electrostatic wakes, and the data affected by wakes need to be excluded from the data set used to determine the offsets.

In the ISR2 offset determination procedure, we decided not to use any reference data, but rather to use a condition of zero electric field $\left\langle E_{x}\right\rangle=0$, as most of the time the electric fields are very weak in the magnetotail ( $X \mathrm{GSE}<0$ and $R>5 \mathrm{RE}$ ), and the average over a tail season must be very close to zero, and the difference from zero gives a rather good 


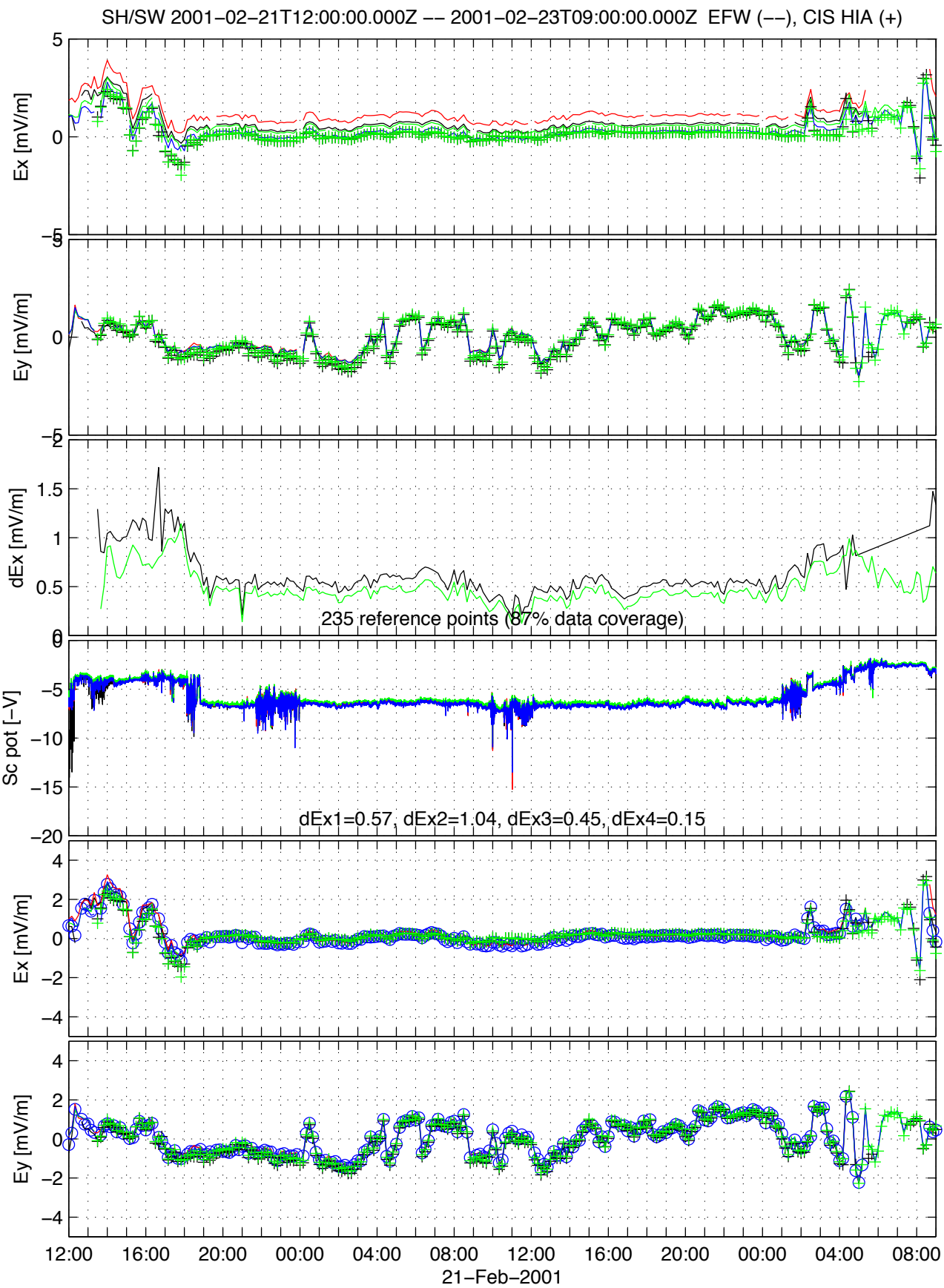

Figure 5. Inter-spacecraft calibration and cross-calibration with CIS in the solar wind/magnetosheath. Panels from top to bottom show $E_{x}$ and $E_{y}$ measured by EFW (solid lines) on the four spacecraft and by CIS-HIA ( + ) on C1 and C3, difference in $E_{x}$ between CIS and EFW (median value from all spacecraft), the spacecraft potential, and the two bottom panels show the same data as on the top, but with the offsets applied to the EFW data. Data from the four Cluster spacecraft shown by black (C1), red (C2), green (C3) and blue (C4).

estimate for the ISR2 $E_{x}$ offset. The resulting offsets were verified against the CIS data for a large number of cases, and in particular in the central plasma sheet the agreement is very good.

Results for Cluster 4 for years 2002-2005 are summarized in Fig. 7. One can see that there is a prominent peak around
$1.3 \mathrm{mV} \mathrm{m}^{-1}$ for all years. However, there is also some group of points giving rise to a broadening towards lower offset values. Therefore we can conclude that the offset value is rather stable, and the broadening is due to actual geophysical electric fields present in the magnetosphere. 


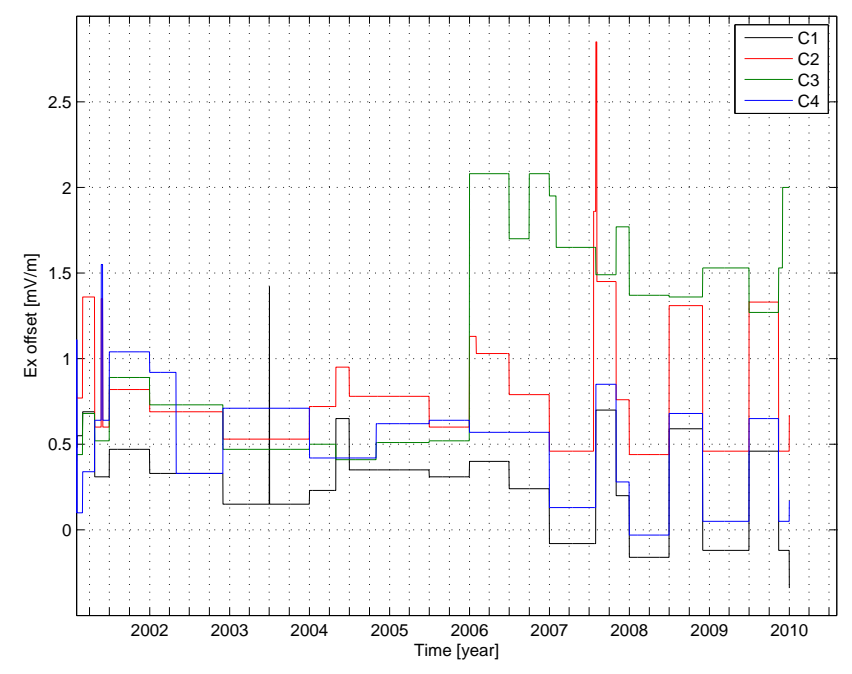

Figure 6. Long-term evolution of the ISR $2 E_{x}$ (sunward) offset in the solar wind/magnetosheath from 2001 to 2009.

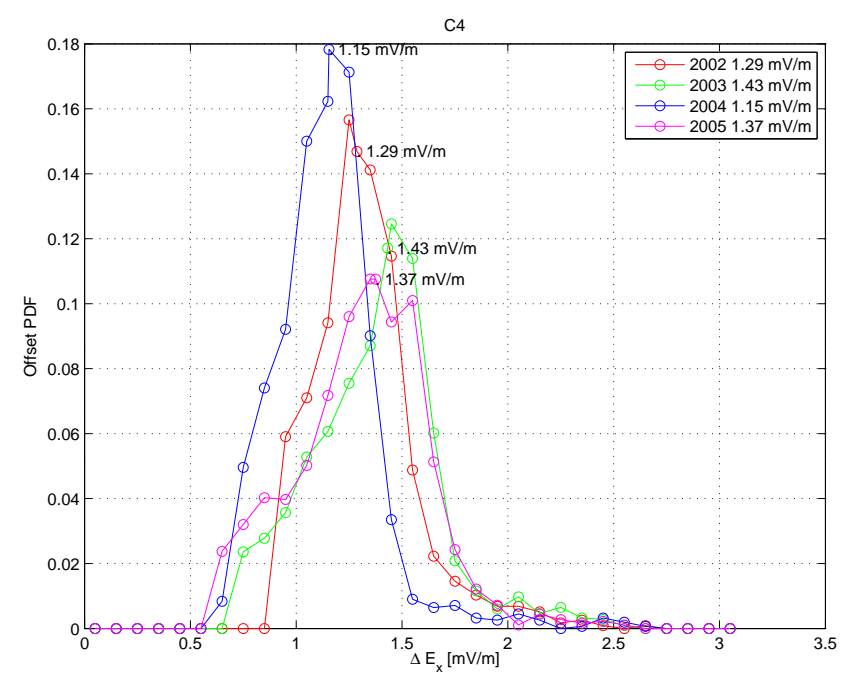

Figure 7. Probability distribution function of ISR $2 E_{x}$ (sunward) offset for Cluster 4 in the magnetosphere for 2002-2005.

Evolution of ISR2 offsets in the magnetosphere during mission lifetime is shown in Fig. 8. The offsets are steady and slowly decreasing with approach of the solar minimum.

\subsection{Delta offset}

Given the two identical probe pairs, we are able to estimate the electric field at the timescale of the spacecraft spin from each of them, and in principle these estimates should be identical. In reality the probes are not identical, and the estimates of the electric fields differ. Such a difference is described by the delta offset. Figure 9 shows how the delta offsets change over time. The curves show the raw data, i.e., the difference between the electric fields computed from the two probe pairs averaged over $1.5 \mathrm{~h}$ long intervals of data. One can see

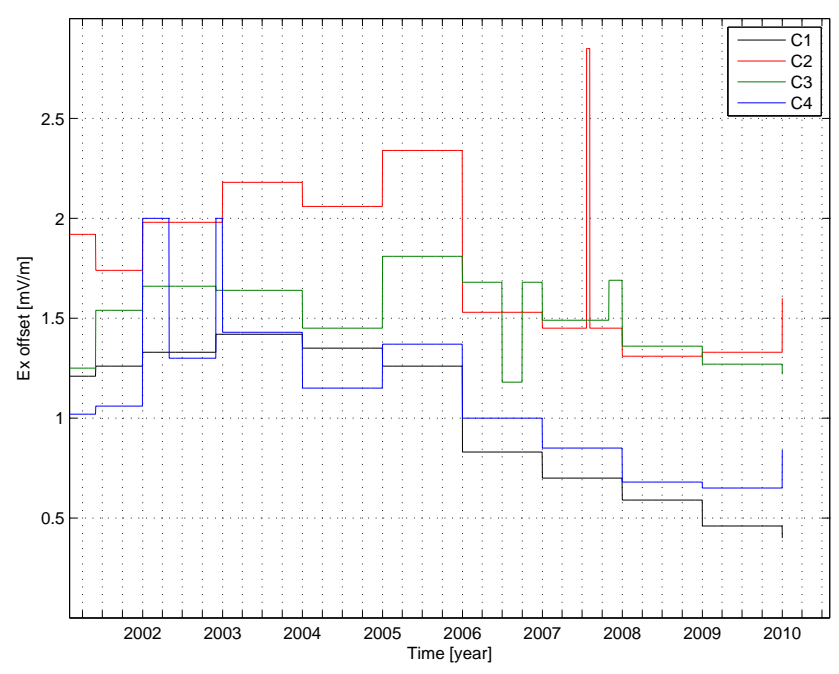

Figure 8. Long-term evolution of the ISR2 offsets in the magnetosphere from 2001 to 2009.

that the offset varies very slowly, at a typical timescale of several months, and with some sudden jumps typically related to spacecraft manoeuvres. Therefore for the calibration purposes we use a smoothened version of the offset, i.e., median over approximately two orbits. This approach allows us to get rid of the outliers, which can be caused by intervals with non-optimal instrument performance or strong geophysical electric fields.

Figure 10 shows the long-term evolution of the delta offsets for all four spacecraft. Variations in the offset are caused by a number of factors. First is the solar cycle. One can see that the offset is rather small and steady in the beginning of the mission and starts to grow with approach of the solar minimum, reaching its maximum in spring 2006. This behavior is caused by non-optimal bias current settings, and the situation became significantly improved by lowering the bias current in June 2006. The second cause is the probe failures, which forced usage of P32 (shorter base and asymmetric with respect to the spacecraft) instead of P12 (see Fig. 1).

\section{Discussion and conclusions}

Here we presented an overview of the calibration procedure used for the DC electric field measurements by EFW on Cluster, which is applied for production of the data for the Cluster Active Archive (CAA). EFW measures potential difference between probes mounted on long wire booms, which, after some corrections, can be used to construct the electric field in the spacecraft spin plane. We show that the calibration procedure leading to an estimate of the geophysical electric field can be described by a set of offsets, which are determined from the symmetry considerations enabled by rapid spacecraft spin as well as from statistical comparisons with other measurements of the electric field on Cluster. We show 


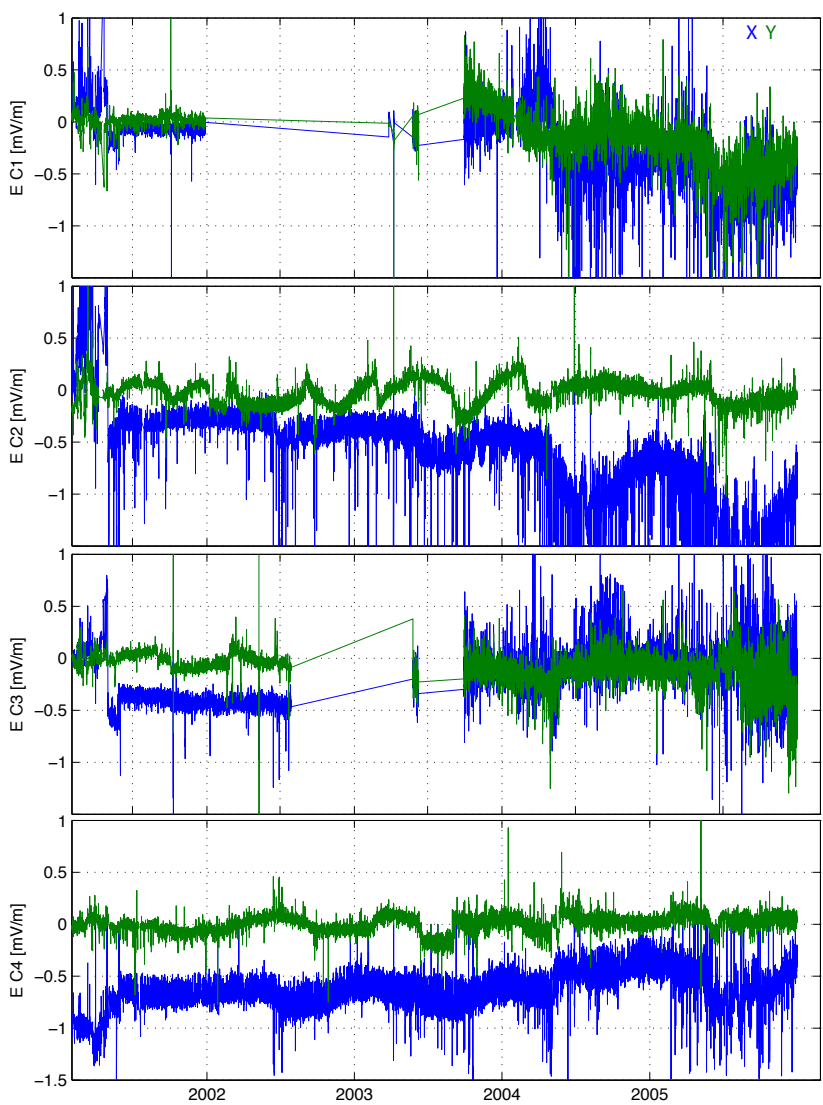

Figure 9. Evolution of the "raw" delta offsets on Cluster 1-4 from 2001 to 2005. The blue and green lines show $X$ and $Y$ components of the difference between the electric fields computed from the different probe pairs, i.e., the "raw" delta offsets. In order to get rid of the outliers (spikes), we compute the median of this difference, which is then used as the delta offset applied to data during the calibration process.

that most of the offsets have a rather slow variation with time on time-space timescales of weeks and month.

Both the amplitude factor (boom shortening) and the sunward offset depend on the Debye length and the spacecraft potential. For the Debye length shorter than the probe-topuck distance ( $1.5 \mathrm{~m}$ for EFW), there is no boom shortening (i.e., amplitude factor $=1$ ); for the longer Debye length the effective boom length is shorter than the physical length, and such a shortening in some way depends on the spacecraft potential. We were unable to establish an empirical relation of the shortening factor with the spacecraft potential for EFW and used a constant amplitude factor for the CAA calibration as the measurement is performed in the long Debye length regime most of the time. We note that even determination of the Debye length on a routine basis is a challenging task for Cluster, due to high uncertainties and insufficient time resolution of the electron data.

Factors determining the sunward offset are much less understood. Simulations by Cully et al. (2007) suggested that

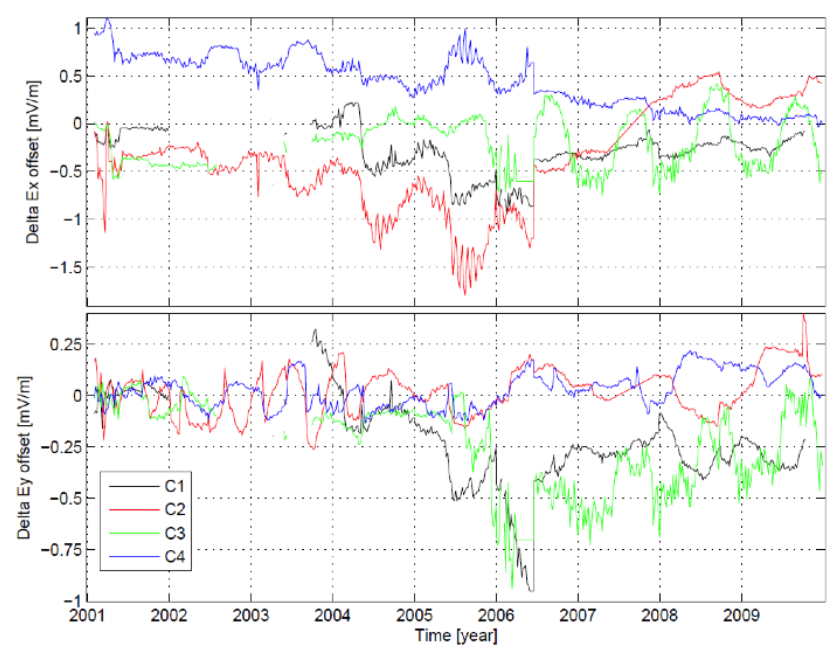

Figure 10. Long-term evolution of delta offsets from 2001 to 2009 for C1 (black), C2 (red), C3 (green) and C4 (blue).

the offset appears due to an asymmetric photoelectron cloud around the spacecraft. However, analyzing changes in the sunward offset for EFW we found that the offset may be strongly driven by other factors, not only for the photoelectron cloud asymmetry. For example the offset in the solar wind depends on the solar wind speed in such a way that it decreases with an increase of the wind speed, and for sufficiently fast solar wind the offset turns into anti-sunward. Qualitative and quantitative understanding of the dependence of the amplitude factor and sunward offset on the surrounding plasma remains an open question, which will be addressed in the future by advanced numerical simulations and possibly by empirical comparison of the electric field measurements by the double probes to EDI and particle instruments on Magnetospheric Multiscale (MMS) Mission.

Using particle measurements to determine time-dependent offsets and to correct the EFW data on a routine basis showed to be practically impossible for Cluster, as such a correction would typically introduce a large random error into the electric field measurement. However, we do no exclude a possibility for such a correction for case studies where one has full control of the quality of the reference data used to determine the offsets. Our choice of the calibration procedure for the CAA which is outlined in this paper is to rely solely on the EFW data for high-time-resolution calibrations, and then to use statistical offsets determined by averaging large amounts of data, and among others by comparison with the particle measurements.

We should note that the presented approach is not capable of correcting for fast changes of the offsets due to rapid crossing of plasma boundaries such as the bow shock and magnetopause. At present, calibration of such events usually requires manual calibration in order to achieve precision on the DC electric field below $1 \mathrm{mV} \mathrm{m}^{-1}$, and reliable calibration is only possible when all probes respond to changes in 
the plasma environment in a very similar way, and the effect of these changes on offsets is not too drastic. Developing a procedure that would produce reliable results also for such cases during routine data production remains a challenging task.

As a concluding remark, we note that the described calibration procedure applies to data acquired when the instrument operates close its optimal regime, so that one can reconstruct the ambient electric field present in the plasma by applying relatively small corrections. However, a major effort during the CAA production goes into detection of strong deviations from the nominal operations (Khotyaintsev et al., 2010), which can be caused by both changes of the plasma environment surrounding the spacecraft and non-optimal instrument settings.

Acknowledgements. The authors thank Andris Vaivads and other colleagues for continuing support and discussion around the coffee breaks.

Edited by: H. Laakso

\section{References}

André, M. and Cully, C. M.: Low-energy ions: a previously hidden solar system particle population, Geophys. Res. Lett., 39, L03101, doi:10.1029/2011GL050242, 2012.

Cully, C. M., Ergun, R. E., and Eriksson, A. I.: Electrostatic structure around spacecraft in tenuous plasmas, J. Geophys. Res., 112, A09211, doi:10.1029/2007JA012269, 2007.

Engwall, E., Eriksson, A. I., André, M., Dandouras, I., Paschmann, G., Quinn, J., and Torkar, K.: Low-energy (order $10 \mathrm{eV}$ ) ion flow in the magnetotail lobes inferred from spacecraft wake observations, Geophys. Res. Lett., 33, L06110, doi:10.1029/2005GL025179, 2006.

Engwall, E., Eriksson, A. I., Cully, C. M., André, M., Torbert, R., and Vaith, H.: Earth's ionospheric outflow dominated by hidden cold plasma, Nat. Geosci., 2, 24-27, 2009a.

Engwall, E., Eriksson, A. I., Cully, C. M., André, M., Puhl-Quinn, P. A., Vaith, H., and Torbert, R.: Survey of cold ionospheric outflows in the magnetotail, Ann. Geophys., 27, 3185-3201, doi:10.5194/angeo-27-3185-2009, 2009b.

Eriksson, A. I., André, M., Klecker, B., Laakso, H., Lindqvist, P.-A., Mozer, F., Paschmann, G., Pedersen, A., Quinn, J., Torbert, R., Torkar, K., and Vaith, H.: Electric field measurements on Cluster: comparing the double-probe and electron drift techniques, Ann. Geophys., 24, 275-289, doi:10.5194/angeo-24-275-2006, 2006.

Eriksson, A. I., Khotyaintsev, Y., and Lindqvist, P.-A.: Spacecraft wakes in the solar wind, in: Proceedings of the 10th Spacecraft Charging Technology Conference (SCTC-10), available at: http://www.space.irfu.se/aie/publ/Eriksson2007b.pdf (last access: 30 January 2014), 2007.

Gustafsson, G., Boström, R., Holback, B., Holmgren, G., Lundgren, A., Stasiewicz, K., Åhlén, L., Mozer, F. S., Pankow, D., Harvey, P., Berg, P., Ulrich, R., Pedersen, A., Schmidt, R., Butler, A., Fransen, A. W. C., Klinge, D., Thomsen, M., Fälthammar, C.-G., Lindqvist, P.-A., Christenson, S., Holtet, J.,
Lybekk, B., Sten, T. A., Tanskanen, P., Lappalainen, K., and Wygant, J.: The Electric Field and Wave Experiment for the Cluster Mission, Space Sci. Rev., 79, 137-156, 1997.

Gustafsson, G., André, M., Carozzi, T., Eriksson, A. I., Fälthammar, C.-G., Grard, R., Holmgren, G., Holtet, J. A., Ivchenko, N., Karlsson, T., Khotyaintsev, Y., Klimov, S., Laakso, H., Lindqvist, P.-A., Lybekk, B., Marklund, G., Mozer, F., Mursula, K., Pedersen, A., Popielawska, B., Savin, S., Stasiewicz, K., Tanskanen, P., Vaivads, A., and Wahlund, J.-E.: First results of electric field and density observations by Cluster EFW based on initial months of operation, Ann. Geophys., 19, 1219-1240, doi:10.5194/angeo19-1219-2001, 2001.

Khotyaintsev, Y., Lindqvist, P.-A., Eriksson, A. I., and André, M.: The EFW Data in the CAA, the Cluster Active Archive, Studying the Earth's Space Plasma Environment, in: Astrophysics and Space Science Proceedings, edited by: Laakso, H., Taylor, M. G. T. T., and Escoubet, C. P., Springer, Berlin, 97-108, 2010.

Lindqvist, P.-A., Cully, C. M., and Khotyaintsev, Y.: User Guide to the EFW measurements in the Cluster Active Archive (CAA), available at: http://caa.estec.esa.int/caa/ug_cr_icd.xml (last access: 30 January 2014), 2013.

Paschmann, G., Melzner, F., Frenzel, R., Vaith, H., Parigger, P., Pagel, U., Bauer, O., Haerendel, G., Baumjohann, W., Sckopke, N., Torbert, R., Briggs, B., Chan, J., Lynch, K., Morey, K., Quinn, J., Simpson, D., Young, C., McIlwain, C., Fillius, W., Kerr, S., Mahieu, R., and Whipple, E.: The electron drift instrument for cluster, Space Sci. Rev., 79, 233-269, 1997.

Paschmann, G., Quinn, J. M., Torbert, R. B., Vaith, H., McIlwain, C. E., Haerendel, G., Bauer, O. H., Bauer, T., Baumjohann, W., Fillius, W., Förster, M., Frey, S., Georgescu, E., Kerr, S. S., Kletzing, C. A., Matsui, H., Puhl-Quinn, P., and Whipple, E. C.: The Electron Drift Instrument on Cluster: overview of first results, Ann. Geophys., 19, 1273-1288, doi:10.5194/angeo-191273-2001, 2001.

Pedersen, A., Mozer, F., and Gustafsson, G.: Electric field measurements in a tenuous plasma with spherical double probes, in: Measurement Techniques in Space Plasmas - Fields: Geophysical Monograph 103, edited by: Pfaff, R. F., Borovsky, J. E., and Young, D. T., published by the American Geophysical Union, Washington, D.C., USA, 1-12, 1998.

Rème, H., Aoustin, C., Bosqued, J. M., Dandouras, I., Lavraud, B., Sauvaud, J. A., Barthe, A., Bouyssou, J., Camus, Th., Coeur-Joly, O., Cros, A., Cuvilo, J., Ducay, F., Garbarowitz, Y., Medale, J. L., Penou, E., Perrier, H., Romefort, D., Rouzaud, J., Vallat, C., Alcaydé, D., Jacquey, C., Mazelle, C., d'Uston, C., Möbius, E., Kistler, L. M., Crocker, K., Granoff, M., Mouikis, C., Popecki, M., Vosbury, M., Klecker, B., Hovestadt, D., Kucharek, H., Kuenneth, E., Paschmann, G., Scholer, M., Sckopke, N., Seidenschwang, E., Carlson, C. W., Curtis, D. W., Ingraham, C., Lin, R. P., McFadden, J. P., Parks, G. K., Phan, T., Formisano, V., Amata, E., Bavassano-Cattaneo, M. B., Baldetti, P., Bruno, R., Chionchio, G., Di Lellis, A., Marcucci, M. F., Pallocchia, G., Korth, A., Daly, P. W., Graeve, B., Rosenbauer, H., Vasyliunas, V., McCarthy, M., Wilber, M., Eliasson, L., Lundin, R., Olsen, S., Shelley, E. G., Fuselier, S., Ghielmetti, A. G., Lennartsson, W., Escoubet, C. P., Balsiger, H., Friedel, R., Cao, J.-B., Kovrazhkin, R. A., Papamastorakis, I., Pellat, R., Scudder, J., and Sonnerup, B.: First multispacecraft ion measurements in and near the Earth's 
magnetosphere with the identical Cluster ion spectrometry (CIS) experiment, Ann. Geophys., 19, 1303-1354, doi:10.5194/angeo19-1303-2001, 2001.
Shue, J.-H., Chao, J. K., Fu, H. C., Russell, C. T., Song, P., Khurana, K. K., and Singer, H. J.: A new functional form to study the solar wind control of the magnetopause size and shape, J. Geophys. Res., 102, 9497-9511, doi:10.1029/97JA00196, 1997. 